Canadian Science Publishing

Biochemistry and Cell Biology Biochimie et biologie cellulaire

\title{
Developmental Role of plk4 in Xenopus laevis and Danio rerio: implications for Seckel Syndrome
}

\begin{tabular}{|r|l|}
\hline Journal: & Biochemistry and Cell Biology \\
\hline Manuscript ID: & bcb-2015-0003.R1 \\
\hline Manuscript Type: & Article \\
\hline Date Submitted by the Author: & $29-A p r-2015$ \\
\hline Keymlete List of Authors: & $\begin{array}{l}\text { Rapchak, Candace; University of Windsor, Biological Sciences } \\
\text { Patel, Neeraj; Western University, Western Centre for Public Health and } \\
\text { Family Medicine } \\
\text { Hudson, John; University of Windsor, Biological Sciences } \\
\text { Crawford, Michael; University of Windsor, Biological Sciences }\end{array}$ \\
\hline \multicolumn{2}{|c}{} \\
\hline
\end{tabular}


Developmental Role of plk4 in Xenopus laevis and Danio rerio: implications for Seckel Syndrome

Candace Elaine Rapchak ${ }^{1}$, Neeraj Patel ${ }^{2}$, John Hudson $^{1 *}$, Michael Crawford ${ }^{1 *}$

${ }^{1}$ Dept. Biological Sciences, University of Windsor, 401 Sunset Ave, Windsor Ontario N9B 3P4, Canada

${ }^{2}$ Western Centre for Public Health and Family Medicine, Western University, London, Ontario, N6A 2B7, Canada

*Equal corresponding authors :jhudson@uwindsor.ca, mcrawfo@uwindsor.ca

Running Header: A role for plk4 in tissue organization? 


\begin{abstract}
The polo-like kinases are a family of conserved serine/threonine kinases that play multiple roles in regulation of the cell cycle. Unlike its four other family members, the role of Plk4 in embryonic development has not been well characterized. In mice, Plk4 ${ }^{-/-}$ embryos arrest at E7.5, just prior to the initiation of somitogenesis. This has led to the hypothesis that Plk4 expression may be essential to somitogenesis. Recently characterized human mutations lead to Seckel Syndrome. Riboprobe in situ hybridization revealed that plk4 is ubiquitously expressed during early stages of development of Xenopus and Danio; in later stages, expression in frogs restricts to somites as well as eye, otic vesicle, and branchial arch, and brain. Expression patterns in fish remain ubiquitous. Both somite and eye development require planar cell polarity, and disruption of plk4 function in frog by means of morpholino-mediated translational knockdown yields orientational disorganization of both these structures. These results provide the first steps in defining a new role for plk4 in organogenesis and implicates a role in planar cell polarity, segmentation, and in recently described PLK4 mutations in human.
\end{abstract}

Key Words: polo like kinase, somite, lens, centrosome, polarity 


\section{Introduction}

Plk4 is a member of the polo-like family of serine-threonine kinases, which contain four other members, $P l k 1 / 2 / 3 /$ and 5. These kinases are best known for their roles in cell cycle regulation and more specifically in mitotic progression and centrosome duplication (Sunkel and Glover 1988; Hudson et al. 2001; Bettencourt-Dias et al. 2005; Habedanck et al. 2005; Ko et al. 2005; Archambault and Glover 2009; Rosario et al. 2010).

Saccharomyces cervisiae, possess only one polo-like gene, cdc5. Drosophila have at least two (polo, sak), while vertebrates such as Xenopus (plk1-5), fish (plk1-4 with 2 versions of paralog 2), mouse (Plk1-5), and human (PLK1-5) have five.

Plk4 is highly expressed in the testes of adult mice as well as in the ovary, spleen, and thymus. During embryogenesis, murine Plk4 is expressed during the proliferative stages of organ development, particularly in the liver, skin, thymus, somites, small intestine and kidney. In addition, Plk4 expression is also seen in less proliferative tissues such as the nasal and olfactory mucosa and respiratory epithelium (Fode et al. 1994).

Mammalian mutants of Plk4 display varied phenotypes. Murine Plk4 null mutants die by day E7.5, just after gastrulation. They lack somites, notochord and neural tube even though neural crest develops (Hudson et al. 2001). Null mutant embryos have a normal population of trophoblast cells, however, the number of differentiated giant cells is reduced, commensurate with a lack of Hand1 localization to the nucleolus (Martindill et al. 2007). The inability of murine embryos to survive post gastrulation indicates a specific role for Plk4 in the stages of development when organs begin to pattern and organize themselves. Both null and heterozygous murine cells exhibit a delay in mitotic progression (Hudson et al. 2001; Ko et al. 2005). In contrast to Plk4-null mice, heterozygote embryos develop normally without any externally obvious morphological phenotypes, however, elderly mice have an increased incidence of spontaneous liver and lung tumours indicating Plk4 is haploinsufficient for tumour suppression. Plk4-null mice survive longer into development than Plkl-null mice which are embryonic lethal at the morula stage (Lu et al. 2008). By contrast, Plk2- null and Plk3-null mice are viable, so perhaps Plk4-null embryos survive through to gastrulation because of redundancy with 
other Plk family members (Swallow et al. 2005).

In humans, mutation of either PLK4, or its interacting partner CEP152, causes Seckel Syndrome, a disorder associated with dwarfism, microcephaly, and abnormal eye, jaw, ear, and sometimes, spinal development (Kalay et al. 2011; Martin et al. 2014; Shaheen et al. 2014). In the case of PLK4, the dysfunction is only partial as the kinase domain remains intact. There are interesting phenotypic differences that can best be explained by understanding the subdomains affected and, we predict, the subcellular localization patterns in these different mutants. Finally, low viability of human blastocysts maps to an SNP in the domain containing PLK4 (McCoy et al. 2015).

In its N-terminal, like all other polo-like kinases, Plk4 encodes a kinase domain responsible for phosphorylating protein substrates, and at the $\mathrm{C}$-terminal, one canonical and two cryptic polo boxes that are thought to mediate protein-protein interactions and cellular localization (Leung et al. 2007). To date, Plk4 has been characterized predominantly with regard to its importance in centrosome duplication: aberrant levels of Plk4 protein result in the generation of supernumerary centrosomes and genome instability. For example, in tissue culture, PLK4 over-expression leads to the formation of supernumerary centrioles while loss of PLK4 leads to loss of centrosomes and mitotic defects (Bettencourt-Dias et al. 2005; Habedanck et al. 2005; Ko et al. 2005; KleyleinSohn et al. 2007; Rodrigues-Martins et al. 2007). Additionally, plk4 can induce de novo centrosome formation in both Drosophila and Xenopus (Rodrigues-Martins et al. 2007; Eckerdt et al. 2011). Mutations that affect either its phosphorylation, partner-interacting, or subcellular localization characteristics also have phenotypic effects (Leung et al. 2002; Elia et al. 2003; Habedanck et al. 2005; Leung et al. 2007; Slevin et al. 2012).

PLK4 protein localizes to the centrosome during the cell cycle where it physically interacts with several components of the centrosome biogenesis machinery such as CEP152, CEP192, CDK11 and GCP6 and FBXW5 (Hudson et al. 2001; Leung et al. 2002; Hatch et al. 2010; Franck et al. 2011; Puklowski et al. 2011; Bahtz et al. 2012; Sonnen et al. 2013). Additionally, PLK4 has been shown to phosphorylate p53 and CHK2, both of which are important components of the DNA damage response (Ko et al. 
2005; Petrinac et al. 2009). PLK4's role in other cellular compartments is less clear.

Although murine Plk4 predominately localizes to the centrosome, in G2 of the cell cycle it briefly moves to the nucleolus, an action that coincides with the differentiation of trophoblast stem cells (Hudson et al. 2001; Martindill and Riley 2008). Plk4 directly phosphorylates Hand1 in the nucleolus to influence trophoblast stem cell differentiation during mouse placental development (Martindill et al. 2007).

The lethality of Plk4 null mice makes further study of its role in development difficult using this model system. We undertook to characterize developmental expression of plk4 in Xenopus and Danio. We then used morpholino mediated translational knockdown to study the plk4 loss of function phenotype in frog: we demonstrate a role for plk4 in organizing tissues during organogenesis, and implicate a new function for plk4 in localizing the centrosome to establish cell polarity, and potentially to link mitosis to the segmentation clock/oscillator.

\section{Materials and Methods}

\section{Frogs}

Xenopus laevis females were induced to ovulate with injection of $0.6-0.8 \mathrm{cc}$ of human Chorionic Ganadotrophin hormone (hCGH) (Intervet Canada Corp.). Embryos were obtained via in vitro fertilization and de-jellied in $2 \%$ cysteine $(\mathrm{pH} 8)$ as previously described (Drysdale and Elinson 1991). For wild type analysis embryos were left to develop in 0.1x MBS (88mM NaCl, $1 \mathrm{mM} \mathrm{KCl,} \mathrm{0.7mM} \mathrm{CaCl,} 1 \mathrm{mM} \mathrm{MgSO} 4,5 \mathrm{mM}$ HEPES pH7.8, 2.5mM NaHCO3) at either $12^{\circ} \mathrm{C}$ or $17^{\circ} \mathrm{C}$. Embryos to be used for morpholino injection were always incubated at $12^{\circ} \mathrm{C}$ post-injection. Embryos were staged according to (Nieuwkoop and Faber 1967), fixed in MEMPFA (0.1M MOPS pH 7.4, $1 \mathrm{mM} \mathrm{MgSO} 4,2 \mathrm{mM}$ EDTA, 4\% paraformaldehyde) at $4{ }^{\circ} \mathrm{C}$ overnight, and stored long term in $70 \%$ methanol.

\section{Zebrafish}


Adult zebrafish were organized in breeding systems with a male-to-female ratio of 1:2 in order to maximize spawning. Embryos were collected from the bottom of breeding tanks by siphoning (performed by Dr. Dennis Higgs, University of Windsor, Department of Biology), and then transferred to fresh carbon-treated tap water. Embryos were then dechorionated using Dumont \#5 watchmaker forceps if they had reached a stage after initiation of pigmentation. Otherwise, dechorionation was performed after fixation to preserve embryo integrity. Embryos were fixed with 4\% paraformaldehyde (PFA) dissolved in PBS-Tween overnight at $4{ }^{\circ} \mathrm{C}$. The next day, embryos were rinsed three times in 1x PBS, then dehydrated through an increasing methanol gradient for storage in $100 \%$ methanol at $-20^{\circ} \mathrm{C}$. They were processed for riboprobe in situ hybridization and probed at high stringency with zebrafish plk4 (pBluescript-SKzplk4 from Open Biosystems Inc) sense and antisense probes, in the manner described for frogs embryos

\section{Cloning}

The Xenopus probes were derived from $p C M V$-SPORT6-Plx4 (Open Biosystems Inc.). A plk4 truncation construct containing the first 1200bp of the plk4 cDNA was cloned for riboprobe production. Plk4-Sport6 was digested with HindIII to remove 2354bp from the 3' end of the plk4 cDNA insert. The larger fragment, containing the Sport-6 vector backbone and the first $1200 \mathrm{bp}$ of plk4 cDNA, was then re-ligated to circularize.

\section{In Situ Hybridization}

Staining to detect mRNA localization via in situ hybridization was performed according to standard protocols using digoxigenin-UTP (Roche) labeled probes that were hybridized at high stringency $\left(68^{\circ} \mathrm{C}\right)$ (Sive et al. 2000). Embryos were imaged directly or cleared with BABB (2 parts benzoic acid: 1 part benzyl benzoate) prior to image collection using a Leica MZFLIII stereoscope and Northern Eclipse software (Empix, Canada).

\section{Morpholino Design and Microinjection}


For translational knockdown assays anti-sense morpholino oligonucleotides were designed and ordered through GeneTools, LLC (Orlando, USA). To confirm specificity two plk4 specific morpholinos were designed to target different regions of the plk4 transcript. Plk4.upMO sequence targeting the 5' UTR was 5'TCTTTTTCTCTCGCTGCTCGCGCCC-3' and Plk4.1MO sequence targeting the ATG start site was 5'-CTCTCTCCTATGCTGCCCGCCATGC-3'. The mis-match control sequence corresponding to Plk4.1 was 5'-CTCTCTTCgTATcCTcCCCcCCATcC. A standard control morpholino targeted against the human $\beta$-globin gene was also ordered from GeneTools with sequence 5'-CCTCTTACCTCAGTTACAATTTATA-3'. All morpholinos were tagged with a 3'-Carboxyfluoroscein moiety for lineage tracing. Embryos to be used for morpholino microinjection were incubated briefly in $0.3 \mathrm{x}$ MBS and 2\% Ficoll-400 (Sigma) prior to injection and then microinjected into their animal pole at either the 1 or 2 cell stage. Microinjections were performed using a Drummond nanoinjector maintained at a volume of $4.6 \mathrm{~nL}$. Post-injected embryos were incubated in $0.3 \mathrm{xMBS}$ and $2 \%$ Ficoll- 400 at $12^{\circ} \mathrm{C}$ for at least 1.5 hours to allow healing. Embryos were subsequently moved into $0.1 \mathrm{x}$ MBS for incubation at $12^{\circ} \mathrm{C}$ degrees until they developed to appropriate stages for analysis. Experimental embryos were staged according Nieuwkoop and Faber (1967) and fixed in MEMPFA for either 2 hours at room temperature or overnight at $4^{\circ} \mathrm{C}$.

\section{Immunohistochemistry}

Immunostaining was performed using standard protocols (Sive et al. 2000). Embryos were then incubated overnight at $4^{\circ} \mathrm{C}$ with primary antibodies to either anti- $\beta 1$ integrin (Drs. P. Hausen and V. Gawanta- 8C8, Developmental Studies Hybridoma Bank, Iowa City, USA) at 1:400 dilution, anti-12/101 (Dr. JP. Brockes-12/101, Developmental Studies Hybridoma Bank, Iowa City, USA) at 1:1000 dilution or, anti- $\gamma$ - tubulin (Sigma GTU-88) at 1:100 dilution. Alexa-568 (Life Technologies) secondary antibody diluted 1:500 in PBT was used for $\beta 1$-integrin and $\gamma$-tubulin stained embryos. Anti-mouse-HRP secondary (Sigma) diluted to 1:200 in PBT was used for anti-12/101 embryos. Embryos stained secondarily with Alexa-568 were dehydrated to $70 \%$ methanol for later sectioning. Embryos stained with anti-12/101 were washed twice for 5 minutes with 1x PBS. 
Antibody detection was achieved by addition of DAB (Sigma) diluted to $1 \mathrm{mg} / \mathrm{ml}$ in PBT supplemented with $0.003 \% \mathrm{H}_{2} \mathrm{O}_{2}$. The colour reaction was stopped by two five minute washes with PBT. Embryos were then dehydrated to $70 \%$ methanol and imaged as whole mounts or processed for sectioning and imaging.

\section{Sectioning and Hoechst Stain}

Sections were stained with Hoechst 33342 (Invitrogen) diluted 1:1000 and washed with PBST. Fluorescently stained sections were cover slipped with fluoromount $G$ and imaged. Sectioned riboprobe in situ hybridized specimens that had been sectioned were also imaged on a Zeiss Axioscope fluorescent microscope using Northern Eclipse software.

\section{Results}

\section{Plk4 has a dynamic spatio-temporal pattern of expression during Xenopus development}

While the Xenopus laevis genome has only recently been sequenced, predictive analysis of plk4 indicates a transcript with 16 exons, similar to what is found in mouse and human (mouse - accession NM 011495.2; human - accession NM 001190799.1; Xenopus accession NM 001089677, Xenopus genomic sequence available at xenbase.org). Like its mammalian homologs, Xenopus plk4 encodes both cryptic and the conserved polodomains. One riboprobe was derived from full length $p l k 4 \mathrm{cDNA}$. The second probe was developed using only the first $1200 \mathrm{bp}$ of 5' sequence. Sense riboprobes were developed as controls for both probes.

In Xenopus, plk4 is expressed ubiquitously in the early stages of development compared to sense controls (Fig. 1A-B). Expression begins to concentrate dorso-anteriorly in late gastrula until by stage 14, the anterior neural plate is particularly rich in transcript (Fig. 1C). At around stage 17 the plk4 expression domain becomes more intense, particularly in the anterior neural plate, along the neural ridges, and then in neural tube, pre-somitic mesoderm and lateral plate mesoderm (Fig. 1D). At stage 20, expression restricts to the anterior somites, neural plate, the optic vesicles, and developing brain, so that by stage 24 , when otic vesicle is forming, all these structures express plk4 (Fig 1E-F). Faint 
expression in the pre-somitic mesoderm (PSM) remains throughout the processes of segmentation as well as in newly formed somites (Compare sense control $1 \mathrm{G}$ to $\mathrm{H}$ in stage 30 embryos). In maturing somites, plk4 expresses along the entire anterior-posterior length of the embryo as well as in developing lens and otic vesicle (Fig. 1I). plk4 expression concentrates in somites predominantly dorsally in stage 22 embryos, and with a very slight central bias (Fig. 1J), in a location where the nuclei are seen to align after rotation. When looked at from the top at stage 26, there is still somitic expression (Fig. $1 \mathrm{~K})$. At stage 35, plk4 expresses strongly in the lens vesicle although somewhat less so in developing retina, neural tube, notochord, and branchial arches (Fig. 1L). Sense probes produced a fainter signal than antisense, however it was consistent and present at high stringency and was produced in multiple replicates and hands.

In zebrafish, the gene is ubiquitously expressed from early through late stages of development (Figure 2A-H). Embryos incubated with the sense probe served as a negative control in these experiments, showing only background, non-specific staining compared to antisense-probed embryos.

\section{Depletion of Xenopus plk4 disrupts somite morphogenesis and patterning}

Morpholino specificity was confirmed by four methods: morpholinos directed against two separate 5' sites produced identical results (Fig 3A); neither control mismatch nor standard control morpholinos produced these effects (Fig 3B); low combined concentrations of targeting morpholinos produced phenotypes consistent with the highest concentrations of either one alone (Table 1); plk4 mRNA rescued a morphant eye phenotype (Fig 4). Somite phenotypes are elicited by both plk4 over- as well as underexpression, and the consequent "rescue" combinations of mRNA and morpholino are difficult to interpret without ambiguity. Ectopic expression of Plk4 is known to induce supernumerary centrosomes in both frog and fly (Rodrigues-Martins et al., 2007; Eckerdt et al., 2011), so the competing and divergent effects are not without precedent. It is worth mentioning that some of the phenotypes that survived late into development, when heart normally starts beating, were similar to morphologies produced by off-target effects in fish - but this was expected since plk4 interacts with both hand1 and p53, and would 
produce similar effects if acting as predicted: for example hand 1 mutant mice display heart and craniofacial anomalies (Firulli et al. 1998; Firulli et al. 2014). Additionally, plk4 can induce de novo centrosome formation in both Drosophila and Xenopus (Rodrigues-Martins et al. 2007; Eckerdt et al. 2011). We have never seen this category of morpholino off-target effect in frog. Moreover, the morphologies obtained are consistent with mouse and human mutant phenotypes. We conclude that the action of the morpholino treatment is specific.

Examination of the gross morphology of plk4MO (morpholino) embryos that were unilaterally injected at the 2 cell stage (the uninjected side serves as a control) indicates that depletion of plk4 protein results in a bent dorsal axis (Fig. 3C) and an eye phenotype (compare control Fig. 3D with morphant E). The eye phenotype ranged from diminished size (usually weakening ventrally first; Fig. 3E) to a complete absence of eye, and this latter was also associated with microcephaly. Ventrally diminished retinal pigmentation remained evident through to the late stage $30 \mathrm{~s}$, and this is as far as we followed the phenomena. A marker of lens placode formation, pitx3, can be completely inhibited by morpholino-mediated knockdown of plk4 (Fig. 3F). The morpholino does not appear to inhibit expression of other markers that are expressed in retina, although the expression domains are altered. In the case of pax6, the domain is slightly diminished (Fig. 3G), and with raxl, inhibition in the optic vesicle following the lens placode stage is more severely curtailed (Fig. 3H). Among those embryos where placode is not completely abolished, eye development can still proceed to generate a relatively normal organ (Fig. 3I), albeit somewhat smaller than usual.

High doses of plk4MO (10ng) cause curvature of the dorsal axis that is the product of inhibited somitogenesis. Normally, pre-somitic mesoderm cells line up along the dorsal axis and then rotate $90^{\circ}$ in cohorts of roughly 10-12 cells, however although such perpendicular cohorts emerge on the control side, the nuclei within the somitic mesoderm of the plk4MO injected side are randomly distributed. This is evident whether somitogenesis is monitored in whole mounts using antibody marker for somite myofibres (Fig. 3J, completely abolished in $87 \%$ of embryos), or in sections using Hoechst stain for nuclei (Fig. 3K-L). Control somites differentiate a morphology that is characterized by 
defined borders and striated myofibre cells, however, even though the morphant side ultimately assumes a modicum of organization, somitic borders indicated by beta1integrin remain poorly defined (compare the top control half with the morphant bottom of Fig $3 \mathrm{~K}$ ). Moreover, in morphants, the nuclei in somitic mesoderm do not organize, nor elongate normally (compare control to morphant sides in $3 \mathrm{~K}-\mathrm{O}$ ), and gamma-tubulin stained centrioles fail to associate in a peri-nuclear fashion (compare Fig $3 \mathrm{~N}$ to $\mathrm{O}$ ). A similar failure of centrioles to associate with smaller nuclei was noted in lens placode as well. Embryos injected with $p l k 4 M O$ also appear to demonstrate impaired function of the segmentation clock: both notch and hes 4 banding patterns are inhibited on the morphant side (Fig 3P and Q respectively).

\section{Discussion}

Both PLK4 and its interacting partner, CEP152 have been identified as loci involved in the human disorder Seckel Syndrome (Kalay et al. 2011; Martin et al. 2014; Shaheen et al. 2014). In one instance, the encoded kinase domain remains intact, but the functions of both the cryptic and conserved polo domains, as well as two of three PEST motifs are abrogated (Shaheen et al. 2014). These deficits presumably interfere, at minimum, with the capacity of PLK4 to interact with partners like CEP152. Possibly, turnover of the protein could be impaired as well. In the other human mutation, the creation of an errant splice acceptor causes a missense mutation that interrupts the final and conserved polodomain by premature termination (Martin et al. 2014). In this allele, all three PEST motifs as well as the cryptic polo-domains remain intact. The third and stereotypic polo domain is required for the protein to lose its auto-inhibition, and it is critical to stability and targeting for degradation (Klebba et al. 2015). Intriguingly, in zebrafish treated with morpholinos to inhibit translation of the last exon that contains the polo-box, the fish suffered a phenotype similar to that seen in human: foreshortened bodies, dorsal axis curvature, and reduced eye size (Martin et al. 2014). An additional defect in fish involved cardiac laterality defects. Cardiac defects have also been documented in Seckel patients (Can et al. 2010; Arslan et al. 2014).

Why, if the mammalian PLK4 gene is expressed in a spatio-temporally restricted manner 
and, as we show, fish plk4 is expressed ubiquitously, are the phenotypes so similar? We speculate that both isoforms remain auto-inhibited, but can still bind with localized partners, such as CEP152, through their remaining and intact cryptic polo domain. This kinase-inhibited complex would produce an effect identical to the more severely truncated human mutant gene (Shaheen et al., 2014) that, lacking even the cryptic polo domain, would be unable to bind partners leaving them similarly unphosphorylated.

In our Xenopus study, the expression pattern of plk4 corresponded well with patterns seen in mouse, however fainter antisense patterns posed a problem. There are no obvious repetitive sequences in plk4, but a sense signal would be consistent with reports of lncRNA in mouse Plk family members (Saito et al. 2011). Human PLK4 is a likely site of intense miRNA complementarity - RepTar reports that 65 different miRNAs can bind human PLK4 (Elefant et al. 2011). If present in sufficient quantity, they could bind probe to produce signal: for example, we can detect the short sequences of abundant antisense morpholino by similar means. The phenotypes of morphants are consistent with the lack of somites in null mutant mice, the eye phenotypes in morphant zebrafish (Martin et al. 2014), as well as with the craniofacial, eye, and otic/ear structures in human. So why was there no lethality in the human and zebrafish studies, and why did somitogenesis occur relatively normally in these organisms?

Our data from frog, as well as the null mutant mouse phenotypes are different from the human and zebrafish phentotypes in a substantial respect - morphants/mutants lack the functionality of a kinase domain as well as all three polo-like domains. In the human and zebrafish phenotypes, the kinase domain remains available and it presumably phosphorylates substrates near centrosomes where PLK4 remains localized, or it may sequester binding partners thereby affecting their availability for downstream processes. In mouse and frog, the simultaneous absence of the kinase domain prohibits all other functions that Plk4/plk4 has assumed over the course of evolution: in addition to the features normally associated with Seckel Syndrome, the animals also lose somites, and consequently a host of other structures necessary to embryonic viability. Why?

One important yet understudied function of the centrosome is its role in establishing 
cellular polarity. The establishment of apico-basal polarity (ABP) is an important aspect of embryonic development, particularly in the formation of epithelial and glandular structures. Several changes occur during establishment of this polarity, one of which involves the re-positioning of the centrosome to the apical surface of the cell. In most epithelial cell types this allows for the formation of a non-centrosomal, apical-basal microtubule array and primary cilia (Musch 2004; Satir and Christensen 2007; Tang and Marshall 2012). Polarization of the microtubule array within the apico-basal axis allows for vesicular transport between apical and basal ends of the cell (Musch 2004). The centrosome is polarized to the apical surface during neural, lens, retinal, mammary, intestinal and cochlear development (Musch 2004; Manning et al. 2008; Tang and Marshall 2012). Moreover, PLK4 mutation results in ciliopathies (Martin et al., 2014). Presumably, centrosome polarity is important to other developmental processes that require the coordinated movement of whole cohorts of cells: among these would fall the elongation, orientation, and alignment of cells that is prerequisite to the formation of somites and lens placode.

In both mammals and frog, lens placode formation represents a first step in the induction of the tissues that form the eye. Mutations that inhibit lens placode development in mammals result in smaller eyes or aniridia (Semina et al. 1998; Semina et al. 2000). In frogs, the absence of placode leads to complete abolition of retinal induction and the eye fails to form (Khosrowshahian et al. 2005). Clearly, inhibition of plk4 translation in frogs is sufficient to inhibit lens placode formation (as monitored by expression of pitx3) and has dire consequences for the development of eye.

Although murine and amphibian somitogenesis appear to be affected in a similar manner by $P l k 4 / p l k 4$ inhibition, the usual process of segmentation differs substantially between the two. In amniotes, the somites bud off and epithelialize, whereas in frogs, cells of the presomitic mesoderm align longitudinally along the dorsal axis, and then rotate in cohorts of 10-12 cells to rest perpendicular to the neural tube (Hamilton 1969). In our study of frog somitogenesis, plk4 dysfunction disrupts this alignment of cells both in the presomitic mesoderm as well as in the regions normally filled with maturing somites: centrosomes appear scattered, and nuclei fail to elongate. Other markers of somite 
maturation were also perturbed including the deposition of beta-integrin that separates somites. Taken in conjunction with the lens placode phenotype, this might suggest a role for plk4 in planar cell polarity. Finally, it is interesting to note that not only was somitogenesis mechanically disrupted, but elements of the segmentation clock (notch and hes4) were also impaired: a previous study has linked the genetic network that embodies the segmentation oscillator/clock to the timing of cytokinesis (Delaune et al. 2012), so it will be interesting in future to query the extent to which Plk4 mediates or coordinates segmentation oscillators with cell division.

\section{Acknowledgements}

Thanks are due to: Drs Zuber, Harris, Kintner, and the NIBB/NIG/NBRP Xenopus laevis EST project for the gift of plasmids; Dr. Dennis Higgs for the gift of zebrafish embryos; Drs. P. Hausen and V. Gawanta via the Developmental Studies Hybridoma Bank (Ames Iowa) for antibody. The technical assistance of Dr. A. Ward and Ms A. Malysa are acknowledged. The Natural Sciences and Research Council (NSERC) of Canada is thanked for funding of Discovery Grants to MJC (203459) and JH (298476). Thanks are also due to NSERC and OGS for scholarships to NP and CER.

\section{References}

Archambault V., and Glover D.M. 2009. Polo-like kinases: conservation and divergence in their functions and regulation. Nat Rev Mol Cell Biol 10: 265-275.

Arslan D., Cimen D., Guvenc O., Sert A., Oktay A., and Oran B. 2014. A case of Seckel syndrome with tricuspid atresia. Genet Couns 25: 171-175.

Avidor-Reiss T., and Gopalakrishnan J. 2013. Building a centriole. Curr Opin Cell Biol 25: $72-77$.

Bahtz R., Seidler J., Arnold M., Haselmann-Weiss U., Antony C., Lehmann W.D., and Hoffmann I. 2012. GCP6 is a substrate of Plk4 and required for centriole duplication. J Cell Sci 125: 486-496.

Basto R., Brunk K., Vinadogrova T., Peel N., Franz A., Khodjakov A., and Raff J.W. 2008. Centrosome amplification can initiate tumorigenesis in flies. Cell 133: 1032-1042.

Bettencourt-Dias M., Rodrigues-Martins A., Carpenter L., Riparbelli M., Lehmann L., Gatt M.K., Carmo N., Balloux F., Callaini G., and Glover D.M. 2005. 
SAK/PLK4 is required for centriole duplication and flagella development. Curr Biol 15: 2199-2207.

Can E., Bulbul A., Uslu S., Demirin H., Comert S., Bolat F., and Nuhoglu A. 2010. A case of Seckel syndrome with Tetralogy of Fallot. Genet Couns 21: 49-51.

Delaune E.A., Francois P., Shih N.P., and Amacher S.L. 2012. Single-cell-resolution imaging of the impact of Notch signaling and mitosis on segmentation clock dynamics. Dev Cell 23: 995-1005.

Drysdale T.A., and Elinson R.P. 1991. Development of the Xenopus laevis hatching gland and its relationship to surface ectoderm patterning. Development 111: 469478.

Eckerdt F., Yamamoto T.M., Lewellyn A.L., and Maller J.L. 2011. Identification of a polo-like kinase 4-dependent pathway for de novo centriole formation. Curr Biol 21: 428-432.

Elefant N., Berger A., Shein H., Hofree M., Margalit H., and Altuvia Y. 2011. RepTar: a database of predicted cellular targets of host and viral miRNAs. Nucleic Acids Res 39: D188-194.

Elia A.E., Rellos P., Haire L.F., Chao J.W., Ivins F.J., Hoepker K., Mohammad D., Cantley L.C., Smerdon S.J., and Yaffe M.B. 2003. The molecular basis for phosphodependent substrate targeting and regulation of Plks by the Polo-box domain. Cell 115: 83-95.

Feldman J.L., and Priess J.R. 2012. A role for the centrosome and PAR-3 in the hand-off of MTOC function during epithelial polarization. Curr Biol 22: 575-582.

Firulli A.B., McFadden D.G., Lin Q., Srivastava D., and Olson E.N. 1998. Heart and extra-embryonic mesodermal defects in mouse embryos lacking the bHLH transcription factor Hand1. Nat Genet 18: 266-270.

Firulli B.A., Fuchs R.K., Vincentz J.W., Clouthier D.E., and Firulli A.B. 2014. Hand1 phosphoregulation within the distal arch neural crest is essential for craniofacial morphogenesis. Development 141: 3050-3061.

Fode C., Motro B., Yousefi S., Heffernan M., and Dennis J.W. 1994. Sak, a murine protein-serine/threonine kinase that is related to the Drosophila polo kinase and involved in cell proliferation. Proc Natl Acad Sci U S A 91: 6388-6392.

Franck N., Montembault E., Rome P., Pascal A., Cremet J.Y., and Giet R. 2011. CDK11(p58) is required for centriole duplication and Plk4 recruitment to mitotic centrosomes. PLoS One 6: e14600.

Guernsey D.L., Jiang H., Hussin J., Arnold M., Bouyakdan K., Perry S., BabineauSturk T., Beis J., Dumas N., Evans S.C., Ferguson M., Matsuoka M., Macgillivray C., Nightingale M., Patry L., Rideout A.L., Thomas A., Orr A., Hoffmann I., Michaud J.L., Awadalla P., Meek D.C., Ludman M., and Samuels M.E. 2010. Mutations in centrosomal protein CEP152 in primary microcephaly families linked to MCPH4. Am J Hum Genet 87: 40-51.

Habedanck R., Stierhof Y.D., Wilkinson C.J., and Nigg E.A. 2005. The Polo kinase Plk4 functions in centriole duplication. Nat Cell Biol 7: 1140-1146.

Hamilton L. 1969. The formation of somites in Xenopus. J Embryol Exp Morphol 22: 253-264. 
Hatch E.M., Kulukian A., Holland A.J., Cleveland D.W., and Stearns T. 2010. Cep152 interacts with Plk4 and is required for centriole duplication. J Cell Biol 191: 721729.

Hudson J.W., Kozarova A., Cheung P., Macmillan J.C., Swallow C.J., Cross J.C., and Dennis J.W. 2001. Late mitotic failure in mice lacking Sak, a polo-like kinase. Curr Biol 11: 441-446.

Januschke J., and Gonzalez C. 2010. The interphase microtubule aster is a determinant of asymmetric division orientation in Drosophila neuroblasts. J Cell Biol 188: 693706.

Kalay E., Yigit G., Aslan Y., Brown K.E., Pohl E., Bicknell L.S., Kayserili H., Li Y., Tuysuz B., Nurnberg G., Kiess W., Koegl M., Baessmann I., Buruk K., Toraman B., Kayipmaz S., Kul S., Ikbal M., Turner D.J., Taylor M.S., Aerts J., Scott C., Milstein K., Dollfus H., Wieczorek D., Brunner H.G., Hurles M., Jackson A.P., Rauch A., Nurnberg P., Karaguzel A., and Wollnik B. 2011. CEP152 is a genome maintenance protein disrupted in Seckel syndrome. Nat Genet 43: 23-26.

Khosrowshahian F., Wolanski M., Chang W.Y., Fujiki K., Jacobs L., and Crawford M.J. 2005. Lens and retina formation require expression of Pitx3 in Xenopus prelens ectoderm. Dev Dyn 234: 577-589.

Kim S., and Dynlacht B.D. 2013. Assembling a primary cilium. Curr Opin Cell Biol 25: 506-511.

Klebba J.E., Buster D.W., McLamarrah T.A., Rusan N.M., and Rogers G.C. 2015. Autoinhibition and relief mechanism for Polo-like kinase 4. Proc Natl Acad Sci U S A 112: E657-666.

Kleylein-Sohn J., Westendorf J., Le Clech M., Habedanck R., Stierhof Y.D., and Nigg E.A. 2007. Plk4-induced centriole biogenesis in human cells. Dev Cell 13: 190202.

Ko M.A., Rosario C.O., Hudson J.W., Kulkarni S., Pollett A., Dennis J.W., and Swallow C.J. 2005. Plk4 haploinsufficiency causes mitotic infidelity and carcinogenesis. Nat Genet 37: 883-888.

Leung G.C., Ho C.S., Blasutig I.M., Murphy J.M., and Sicheri F. 2007. Determination of the Plk4/Sak consensus phosphorylation motif using peptide spots arrays. FEBS Lett 581: 77-83.

Leung G.C., Hudson J.W., Kozarova A., Davidson A., Dennis J.W., and Sicheri F. 2002. The Sak polo-box comprises a structural domain sufficient for mitotic subcellular localization. Nat Struct Biol 9: 719-724.

Lu L.Y., Wood J.L., Minter-Dykhouse K., Ye L., Saunders T.L., Yu X., and Chen J. 2008. Polo-like kinase 1 is essential for early embryonic development and tumor suppression. Mol Cell Biol 28: 6870-6876.

Manning J.A., Colussi P.A., Koblar S.A., and Kumar S. 2008. Nedd1 expression as a marker of dynamic centrosomal localization during mouse embryonic development. Histochem Cell Biol 129: 751-764.

Marthiens V., Rujano M.A., Pennetier C., Tessier S., Paul-Gilloteaux P., and Basto R. 2013. Centrosome amplification causes microcephaly. Nat Cell Biol 15: 731-740.

Martin C.A., Ahmad I., Klingseisen A., Hussain M.S., Bicknell L.S., Leitch A., Nurnberg G., Toliat M.R., Murray J.E., Hunt D., Khan F., Ali Z., Tinschert S., 
Ding J., Keith C., Harley M.E., Heyn P., Muller R., Hoffmann I., Daire V.C., Dollfus H., Dupuis L., Bashamboo A., McElreavey K., Kariminejad A., Mendoza-Londono R., Moore A.T., Saggar A., Schlechter C., Weleber R., Thiele H., Altmuller J., Hohne W., Hurles M.E., Noegel A.A., Baig S.M., Nurnberg P., and Jackson A.P. 2014. Mutations in PLK4, encoding a master regulator of centriole biogenesis, cause microcephaly, growth failure and retinopathy. Nat Genet 46: 1283-1292.

Martindill D.M., and Riley P.R. 2008. Cell cycle switch to endocycle: the nucleolus lends a hand. Cell Cycle 7: 17-23.

Martindill D.M., Risebro C.A., Smart N., Franco-Viseras Mdel M., Rosario C.O., Swallow C.J., Dennis J.W., and Riley P.R. 2007. Nucleolar release of Hand1 acts as a molecular switch to determine cell fate. Nat Cell Biol 9: 1131-1141.

McCoy R.C., Demko Z., Ryan A., Banjevic M., Hill M., Sigurjonsson S., Rabinowitz M., Fraser H.B., and Petrov D.A. 2015. Common variants spanning PLK4 are associated with mitotic-origin aneuploidy in human embryos. Science 348: 235 238.

Musch A. 2004. Microtubule organization and function in epithelial cells. Traffic 5: 1-9.

Nieuwkoop P.D., and Faber J. 1967. Normal Table of Xenopus laevis (Daudin). Amsterdam: North Holland Press.

Petrinac S., Ganuelas M.L., Bonni S., Nantais J., and Hudson J.W. 2009. Polo-like kinase 4 phosphorylates Chk2. Cell Cycle 8: 327-329.

Pihan G.A., Wallace J., Zhou Y., and Doxsey S.J. 2003. Centrosome abnormalities and chromosome instability occur together in pre-invasive carcinomas. Cancer Res 63: 1398-1404.

Puklowski A., Homsi Y., Keller D., May M., Chauhan S., Kossatz U., Grunwald V., Kubicka S., Pich A., Manns M.P., Hoffmann I., Gonczy P., and Malek N.P. 2011. The SCF-FBXW5 E3-ubiquitin ligase is regulated by PLK4 and targets HsSAS-6 to control centrosome duplication. Nat Cell Biol 13: 1004-1009.

Rodrigues-Martins A., Riparbelli M., Callaini G., Glover D.M., and Bettencourt-Dias M. 2007. Revisiting the role of the mother centriole in centriole biogenesis. Science 316: 1046-1050.

Rosario C.O., Ko M.A., Haffani Y.Z., Gladdy R.A., Paderova J., Pollett A., Squire J.A., Dennis J.W., and Swallow C.J. 2010. Plk4 is required for cytokinesis and maintenance of chromosomal stability. Proc Natl Acad Sci U S A 107: 6888-6893.

Rusan N.M., and Peifer M. 2007. A role for a novel centrosome cycle in asymmetric cell division. J Cell Biol 177: 13-20.

Rutland J., and de Iongh R.U. 1990. Random ciliary orientation. A cause of respiratory tract disease. N Engl J Med 323: 1681-1684.

Saito R., Kohno K., Okada Y., Osada Y., Numata K., Kohama C., Watanabe K., Nakaoka H., Yamamoto N., Kanai A., Yasue H., Murata S., Abe K., Tomita M., Ohkohchi N., and Kiyosawa H. 2011. Comprehensive expressional analyses of antisense transcripts in colon cancer tissues using artificial antisense probes. BMC Med Genomics 4: 42.

Satir P., and Christensen S.T. 2007. Overview of structure and function of mammalian cilia. Annu Rev Physiol 69: 377-400. 
Semina E.V., Ferrell R.E., Mintz-Hittner H.A., Bitoun P., Alward W.L., Reiter R.S., Funkhauser C., Daack-Hirsch S., and Murray J.C. 1998. A novel homeobox gene PITX3 is mutated in families with autosomal- dominant cataracts and ASMD. Nat Genet 19: 167-170.

Semina E.V., Murray J.C., Reiter R., Hrstka R.F., and Graw J. 2000. Deletion in the promoter region and altered expression of Pitx 3 homeobox gene in aphakia mice. Hum Mol Genet 9: 1575-1585.

Sepich D.S., Usmani M., Pawlicki S., and Solnica-Krezel L. 2011. Wnt/PCP signaling controls intracellular position of MTOCs during gastrulation convergence and extension movements. Development 138: 543-552.

Shaheen R., Al Tala S., Almoisheer A., and Alkuraya F.S. 2014. Mutation in PLK4, encoding a master regulator of centriole formation, defines a novel locus for primordial dwarfism. J Med Genet 51: 814-816.

Sive H., Grainger R.M., and Harland R.M. 2000. Early Development of Xenopus laevis. A Laboratory Outline. Cold Spring Harbor: Cold Spring Harbor Laboratory Press. $338 \mathrm{p}$.

Slevin L.K., Nye J., Pinkerton D.C., Buster D.W., Rogers G.C., and Slep K.C. 2012. The structure of the plk4 cryptic polo box reveals two tandem polo boxes required for centriole duplication. Structure 20: 1905-1917.

Sonnen K.F., Gabryjonczyk A.M., Anselm E., Stierhof Y.D., and Nigg E.A. 2013. Human Cep192 and Cep152 cooperate in Plk4 recruitment and centriole duplication. J Cell Sci 126: 3223-3233.

Sorokin S. 1962. Centrioles and the formation of rudimentary cilia by fibroblasts and smooth muscle cells. J Cell Biol 15: 363-377.

Sunkel C.E., and Glover D.M. 1988. polo, a mitotic mutant of Drosophila displaying abnormal spindle poles. J Cell Sci 89 ( Pt 1): 25-38.

Swallow C.J., Ko M.A., Siddiqui N.U., Hudson J.W., and Dennis J.W. 2005. Sak/Plk4 and mitotic fidelity. Oncogene 24: 306-312.

Tang N., and Marshall W.F. 2012. Centrosome positioning in vertebrate development. J Cell Sci 125: 4951-4961.

Tanos B.E., Yang H.J., Soni R., Wang W.J., Macaluso F.P., Asara J.M., and Tsou M.F. 2013. Centriole distal appendages promote membrane docking, leading to cilia initiation. Genes Dev 27: 163-168.

Wakida N.M., Botvinick E.L., Lin J., and Berns M.W. 2010. An intact centrosome is required for the maintenance of polarization during directional cell migration. PLoS One 5: e15462.

Wang X., Tsai J.W., Imai J.H., Lian W.N., Vallee R.B., and Shi S.H. 2009. Asymmetric centrosome inheritance maintains neural progenitors in the neocortex. Nature 461: 947-955.

Yamashita Y.M., Jones D.L., and Fuller M.T. 2003. Orientation of asymmetric stem cell division by the APC tumor suppressor and centrosome. Science 301: 1547-1550.

Yamashita Y.M., Mahowald A.P., Perlin J.R., and Fuller M.T. 2007. Asymmetric inheritance of mother versus daughter centrosome in stem cell division. Science 315: 518-521.

Yu T.W., Mochida G.H., Tischfield D.J., Sgaier S.K., Flores-Sarnat L., Sergi C.M., Topcu M., McDonald M.T., Barry B.J., Felie J.M., Sunu C., Dobyns W.B., 
Folkerth R.D., Barkovich A.J., and Walsh C.A. 2010. Mutations in WDR62, encoding a centrosome-associated protein, cause microcephaly with simplified gyri and abnormal cortical architecture. Nat Genet 42: 1015-1020.

Zonies S., Motegi F., Hao Y., and Seydoux G. 2010. Symmetry breaking and polarization of the $\mathrm{C}$. elegans zygote by the polarity protein PAR-2. Development 137: 1669-1677. 


\begin{tabular}{|c|c|c|c|c|c|}
\hline & $\begin{array}{l}\text { Phenotype } \\
\& \text { Number }\end{array}$ & $\begin{array}{l}\text { plkMO1 } \\
10 \mathrm{ng}\end{array}$ & $\begin{array}{l}\text { plkMO2 } \\
10 \mathrm{ng}\end{array}$ & $\begin{array}{l}\text { plkMO1 + } \\
\text { plkMO2 } \\
1 \text { ng }\end{array}$ & $\begin{array}{l}\text { CMO } \\
20 \mathrm{ng}\end{array}$ \\
\hline \multirow{4}{*}{$\begin{array}{l}\text { Eyes } \\
\text { Scored } \\
\text { Stages } \\
34-37\end{array}$} & $\mathrm{n}=$ & 44 & 124 & 17 & 143 \\
\hline & Small Eye & $27 \%$ & $25 \%$ & $0 \%$ & $13 \%$ \\
\hline & Dorso/Ventral & $36 \%$ & $25 \%$ & $35 \%$ & $17 \%$ \\
\hline & No Eye & $36 \%$ & $67 \%$ & $65 \%$ & $0 \%$ \\
\hline \multirow{3}{*}{$\begin{array}{l}\text { Somites } \\
\text { Scored } \\
\text { Stages } \\
22-26\end{array}$} & $\mathrm{n}=$ & 54 & 66 & 22 & 174 \\
\hline & Curved Axis & $83 \%$ & $88 \%$ & $100 \%$ & $10 \%$ \\
\hline & $\begin{array}{l}\text { Somites } \\
\text { Inhibited }\end{array}$ & $71 \%$ & $79 \%$ & $91 \%$ & $11 \%$ \\
\hline
\end{tabular}

Table 1 
Table 1. Morpholino phenotype specificity. Data comparing high dose targeting morpholino (10 ng plkMO 1 and 2) phenotypes with low dose combined (1 ng plkMO 1 and 2) and high dose control morpholino experiments (20 ng CMO). 


\section{Figure Legends}

Figure 1. Developmental expression patterns of Xenopus plk4 revealed by riboprobe insitu hybridization. Embryos display ubiquitous expression of plk4 in early blastula (compare sense control A with antisense probe B). Plk4 expression is high in anterior neural plate (ANP) by stage 14 (C), and in anterior neural ridge (ANR) and neural folds (NF) by stage 17 (D). Over the span of early organogenesis (E-F), expression is high in brain (B), neural tube (NT), optic placode (OpP), and otic vesicle (OtV), and subsequently in eye structures, otic placode (OtP), somites (S), and pre-somitic mesoderm (PSM). Stage 30 embryos show expression in somites (S), developing retina (R), lens placode (LP) and branchial arches (compare sense control G to antisense $\mathrm{H}$ ). Although expression remains high in head and somites, plk4 downregulates throughout the rest of stage 33 embryos (I). In cross sections ( $\mathrm{J}$ and $\mathrm{K}$ ), expression localizes to somites (S), and to neural tube (NT), retina (R), lens (L), but is absent from surrounding integument in (L) (bars $40 \mathrm{um})$. 
Figure 2. Zebrafish plk4 is ubiquitously expressed. Riboprobe wholemount in situ hybridization shows expression is ubiquitous at all stages of zebrafish development (compare sense control A,C,E, and G with B, D, F, and H. Darker outlines of developing organs in $\mathrm{H}$ are optical edge effects and are not specific. 
Figure 3. Phenotypes of morpholino-mediated translation knockdown of plk4 in Xenopus. Diagram (A) illustrates placement of the antisense morpholinos relative to the ATG start site of plk4. External morphant morphologies include a bent dorsal axis and eye anomalies (compare controls B and D with morphants $\mathrm{C}$ and $\mathrm{E}$ ). Eye phenotypes ranged from absent, to diminished size, to dorso-ventral patterning defects. Ventral eye development tended to be affected most easily, and this was reflected by abnormal development of pigmented retina (black arrow, Fig. 3 E). Embryos unilaterally injected at the 2 cell stage yield defects detecable by probes for eye development such as pitx 3 in lens placode, pax6 and raxl in developing eye field (black arrow - uninjected side, white arrow - morpholino injected side in F, G and $\mathrm{H}$ respectively). Embryos are picture faceforward from the front. If lens placode was not entirely prohibited, optic induction occurred and produced realtively normal eye structures ( $\mathrm{I}: \mathrm{L}$ - lens, $\mathrm{R}$ - retina). Dorsal curvature resulted from abnormal somite development as revealed by the absence of normal patterns of myogenic markers ( $\mathrm{J}$ : black arrow - uninjected side, white arrow morpholino injected side), or oriented cohorts of pre-somitic cells (compare hoechst stained nuclei in uninjected side - white arrows, compared to disorganized cells below in $\mathrm{K}$ ), and partitioning of somites is clear and discrete as indicated by borders staining red (arrows) for $\beta 1$ integrin on the control side, but is rudimentary, if at all present, on the morphant side $(\mathrm{K})$. At lower magnification the Hoechst-stained nuclei are neatly aligned (white arrows) and cells on the morphant side more obviously disorganized (L) and scattered. In presomitic mesoderm the alignment and stacking of elongate nuclei is ordered on the control side verses the morphant side where nuclei are smaller and fall out of alignment (compare upper control boxed nuclei in $(\mathrm{M})$ to lower boxed morphants). At higher magnification, immunohistochemistry reveals that gamma-tubulin labelled centrioles (arrows) associate with ordered files of elongated nuclei in control somites $(\mathrm{N})$ but among smaller, disordered nuclei on the morphant side (O) (bars - $20 \mathrm{um}$ ).

Segementation clock markers notch and hes 4 are both inhibited by plk4 dysfunction (black arrow - uninjected side, white arrow - morpholino injected side in P and Q). 


\section{Figure 4. Graph of lens phenotypes and rescue of morpholino phentype by} coinjection with plk4 mRNA. Eye placode presence and morhpology was assessed using in situ hybridization of marker pitx3. Severe morpholino phenotypes (lens absent) could be partially rescued by co-injection of plk4 mRNA with morpholino. This pushed more eyes from the "absent" category into the "small lens" or "normal" categories.

Figure represents summed data of experiments in triplicate. SCMO is control morpholino. $* \mathrm{p} \geq .001$ difference between $10 \mathrm{ng}$ dose morpholino alone and $10 \mathrm{ng}$ morpholino plus rescue mRNA. ${ }^{* *} \mathrm{p} \geq .001$ difference between $5 \mathrm{ng}$ dose morpholino and $10 \mathrm{ng}$ morpholino plus rescue mRNA. 

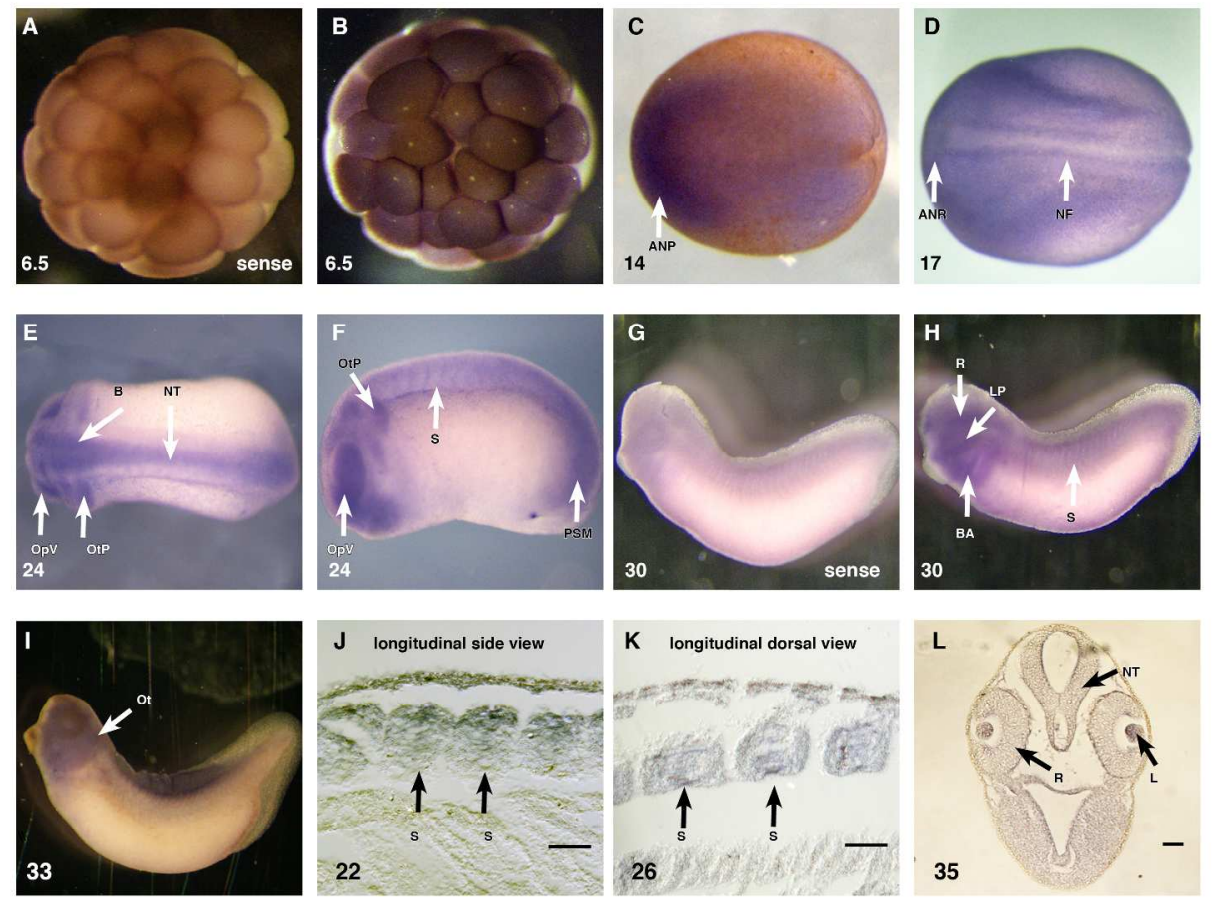

Developmental expression patterns of Xenopus plk4 revealed by riboprobe insitu hybridization. Embryos display ubiquitous expression of plk4 in early blastula (compare sense control A with antisense probe B). Plk4 expression is high in anterior neural plate (ANP) by stage 14 (C), and in anterior neural ridge (ANR) and neural folds (NF) by stage 17 (D). Over the span of early organogenesis (E-F), expression is high in brain (B), neural tube (NT), optic placode (OpP), and otic vesicle (OtV), and subsequently in eye structures, otic placode (OtP), somites (S), and pre-somitic mesoderm (PSM). Stage 30 embryos show expression in somites (S), developing retina (R), lens placode (LP) and branchial arches (compare sense control $G$ to antisense $\mathrm{H}$ ). Although expression remains high in head and somites, plk4 downregulates throughout the rest of stage 33 embryos (I). In cross sections ( $\mathrm{J}$ and $\mathrm{K}$ ), expression localizes to somites (S), and to neural tube (NT), retina $(R)$, lens (L), but is absent from surrounding integument in (L) (bars $40 \mathrm{um}$ ).

$279 \times 215 \mathrm{~mm}(300 \times 300 \mathrm{DPI})$ 


\section{Page 27 of 29}
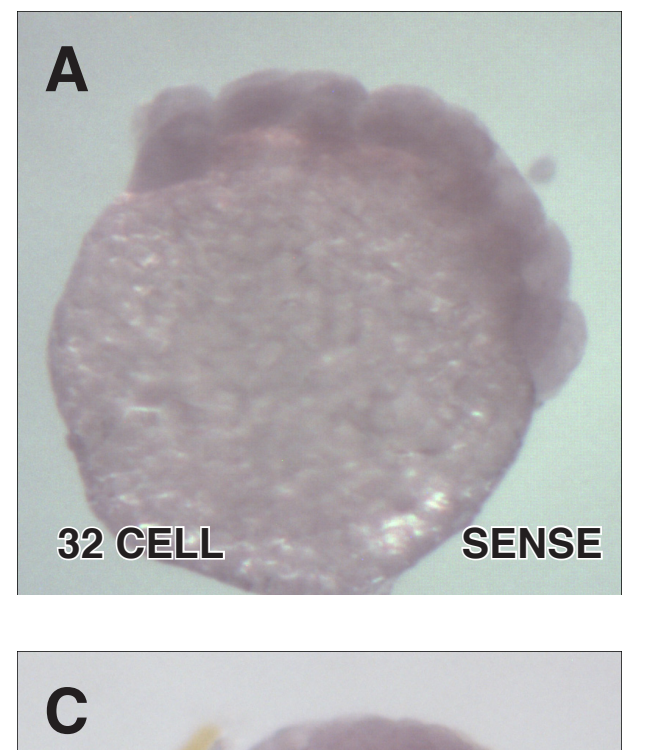

256 CELL

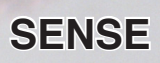

\section{E}

10-13 SOMITE

\section{SENSE}

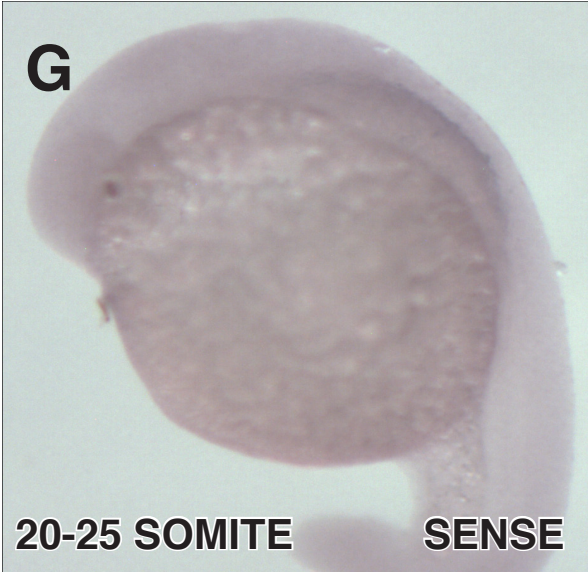

\section{Biochemistry and Cell Biology}
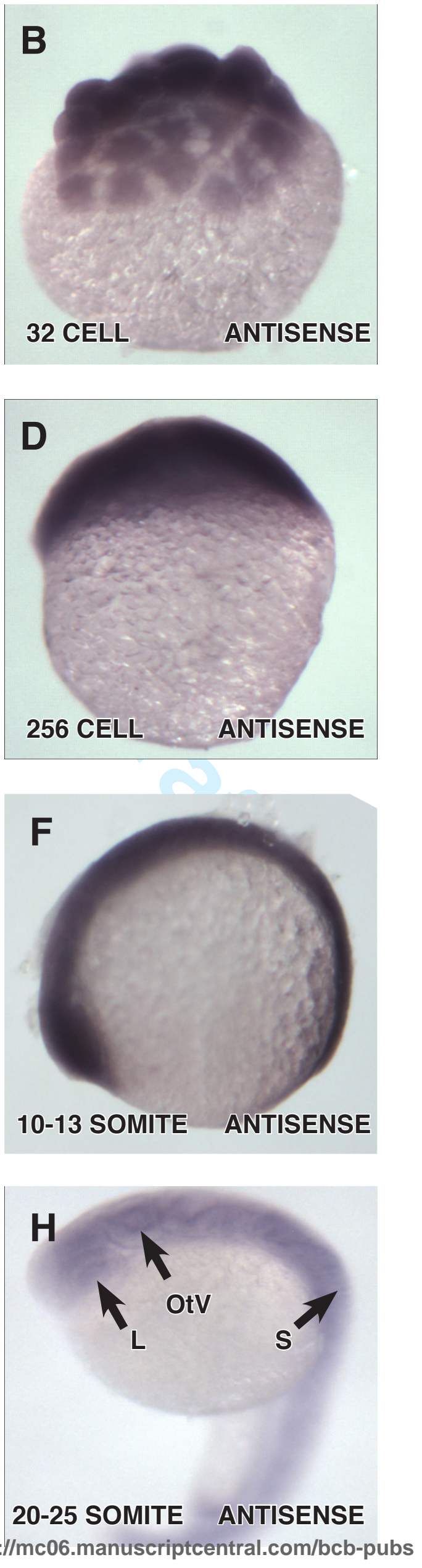

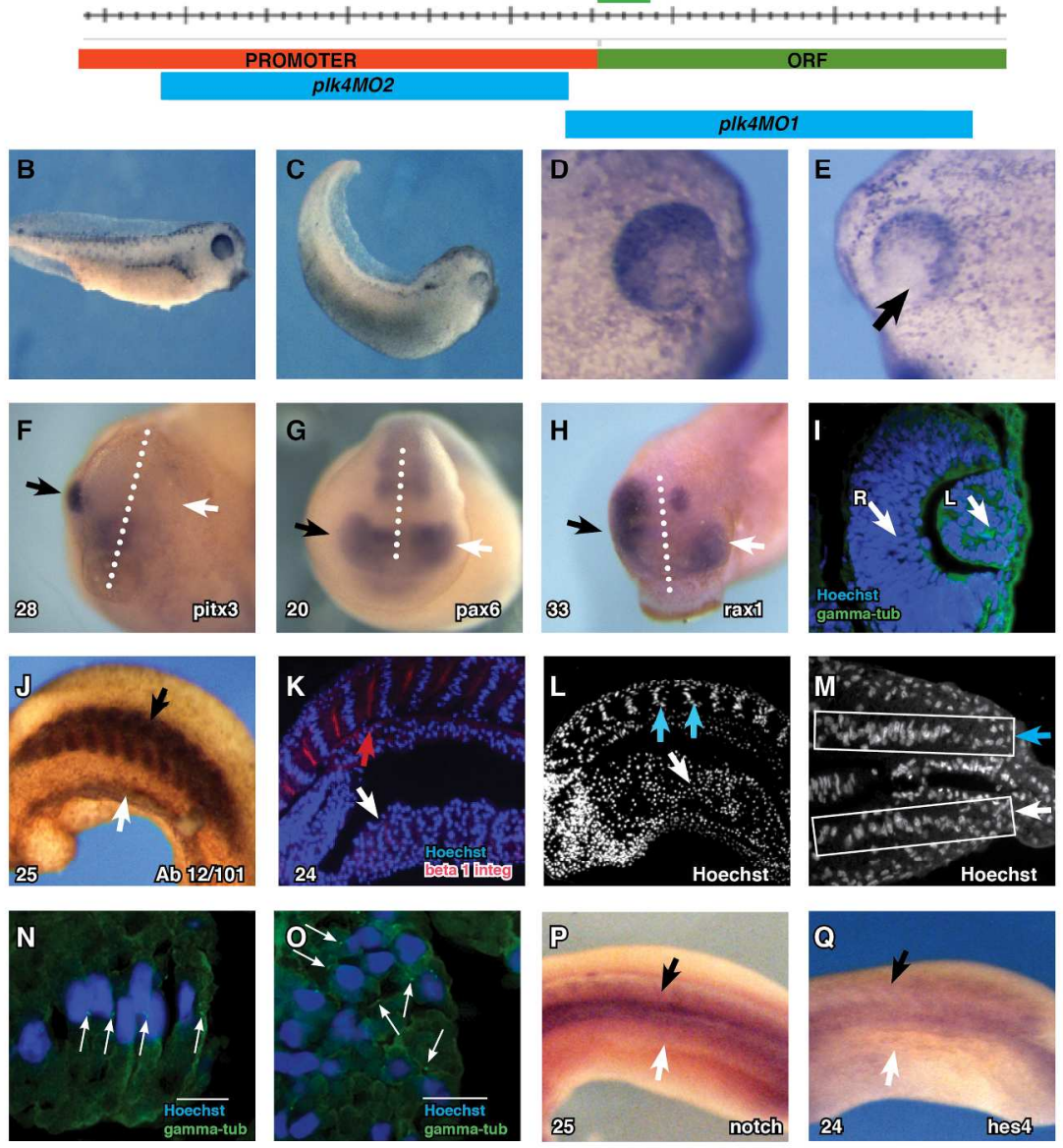

Phenotypes of morpholino-mediated translation knockdown of plk4 in Xenopus. Diagram (A) illustrates placement of the antisense morpholinos relative to the ATG start site of plk4. External morphant morphologies include a bent dorsal axis and eye anomalies (compare controls B and D with morphants $C$ and E). Eye phenotypes ranged from absent, to diminished size, to dorso-ventral patterning defects. Ventral eye development tended to be affected most easily, and this was reflected by abnormal development of pigmented retina (black arrow, Fig. $3 \mathrm{E}$ ). Embryos unilaterally injected at the 2 cell stage yield defects detecable by probes for eye development such as pitx 3 in lens placode, pax 6 and rax 1 in developing eye field (black arrow - uninjected side, white arrow - morpholino injected side in F, G and $\mathrm{H}$ respectively). Embryos are picture face-forward from the front. If lens placode was not entirely prohibited, optic induction occurred and produced realtively normal eye structures (I: L - lens, R - retina). Dorsal curvature resulted from abnormal somite development as revealed by the absence of normal patterns of myogenic markers ( $\mathrm{J}$ : black arrow - uninjected side, white arrow - morpholino injected side), or oriented cohorts of pre-somitic cells (compare hoechst stained nuclei in uninjected side - white arrows, compared to disorganized cells 
below in K), and partitioning of somites is clear and discrete as indicated by borders staining red (arrows) for $\beta 1$ integrin on the control side, but is rudimentary, if at all present, on the morphant side (K). At lower magnification the Hoechst-stained nuclei are neatly aligned (white arrows) and cells on the morphant side more obviously disorganized ( $L$ ) and scattered. In presomitic mesoderm the alignment and stacking of elongate nuclei is ordered on the control side verses the morphant side where nuclei are smaller and fall out of alignment (compare upper control boxed nuclei in ( $\mathrm{M}$ ) to lower boxed morphants). At higher magnification, immunohistochemistry reveals that gamma-tubulin labelled centrioles (arrows) associate with ordered files of elongated nuclei in control somites $(\mathrm{N})$ but among smaller, disordered nuclei on the morphant side (O) (bars - $20 \mathrm{um}$ ). Segementation clock markers notch and hes4 are both inhibited by plk4 dysfunction (black arrow - uninjected side, white arrow - morpholino injected side in $\mathrm{P}$ and $\mathrm{Q}$ ). $215 \times 279 \mathrm{~mm}(300 \times 300 \mathrm{DPI})$ 


\section{Biochemistry and Cell Biology \\ Lens Phenotype (pitx3 ISH)}

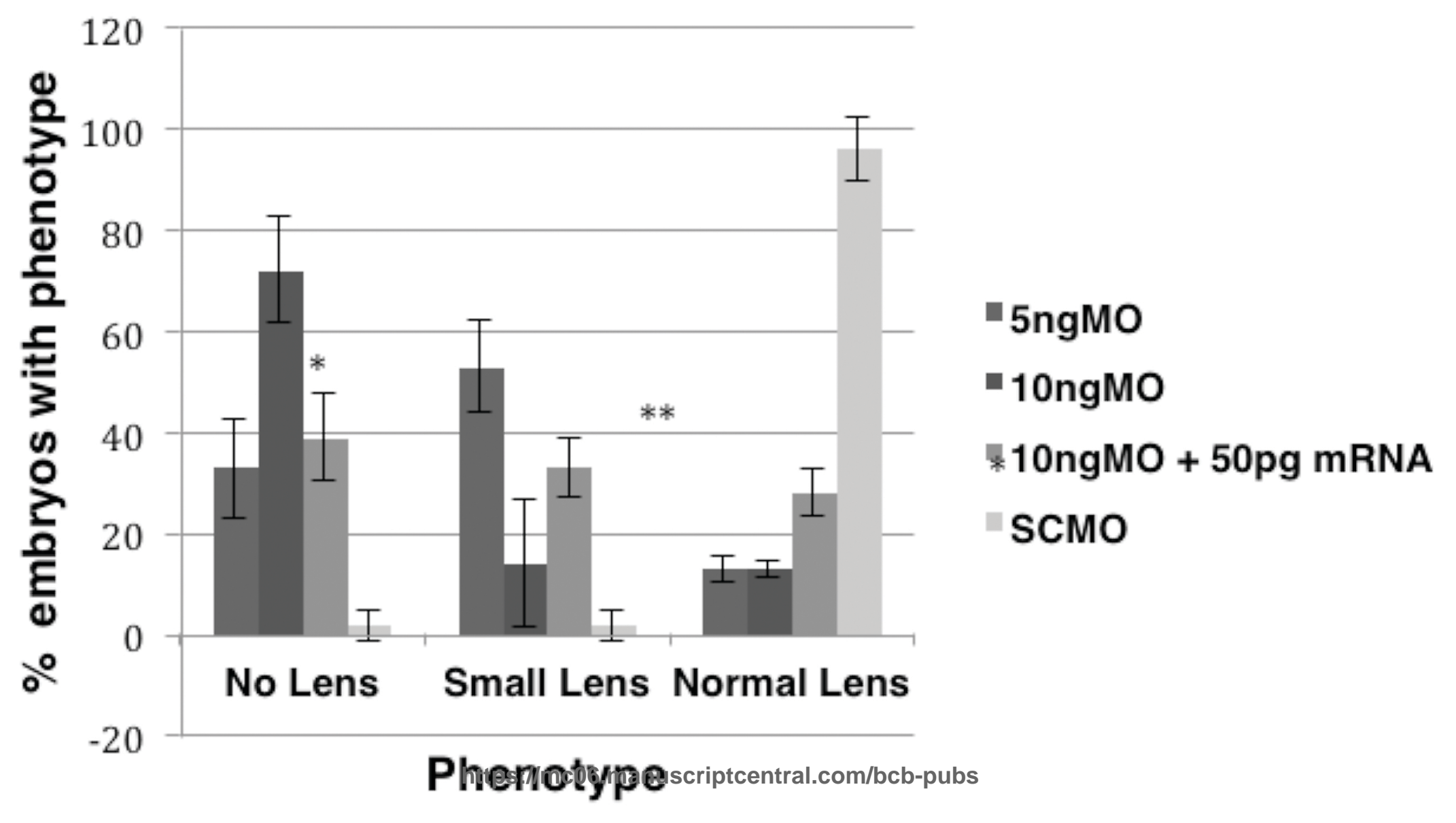

